\title{
INFLUENCIA DE LA TEMPERATURA EN LA FLORACIÓN DE QUERCUS EN EL SUR DE GALICIA (OURENSE Y VIGO, 1994-98)
}

\author{
F. Javier RODRÍGUEZ-RAJO, José MÉNDEZ y María Victoria JATO
}

\begin{abstract}
RESUMEN. Influencia de la temperatura en la floración de Quercus en el sur de Galica (Ourense y Vigo, 1994-98). Los requerimientos necesarios para que las plantas sobrevivan a condiciones climáticas adversas son satisfechos mediante el uso de adaptaciones fisiológicas. Así, las plantas leñosas de la zona templada, para prevenir el congelamiento del agua intracelular, ralentizan su crecimiento hasta casi paralizarlo, entrando en un estado fisiológico denominado "dormancia". Muchos autores indican que el final de la fase de dormancia requiere un período de bajas temperaturas seguido de otro con temperaturas más altas. En el momento en el que los acúmulos de frío y de calor son completados, se produce la floración. El objetivo de este estudio es conocer la influencia que un parámetro climático como la temperatura ejerce sobre Quercus en el sur de Galicia, para poder predecir el inicio del período de dormancia, su duración y los consecuentes requerimientos de frío y calor, para que la floración tenga lugar. En este sentido hemos elegido dos zonas con parámetros de temperatura diferentes: una zona con temperaturas suaves, Vigo y otra con temperaturas mas frías, Ourense. El monitorizaje ha sido llevado a cabo mediante el uso de un captador volumétrico tipo Hirst LANZONI VPPS 2000, de recambio semanal. Quercus comienza su período de polinación en el mes de marzo, se inicia durante la primera quincena en Vigo, y unos pocos días más tarde se produce en Ourense. Durante los cuatro años de estudio, para superar el período de dormancia Ourense necesitó una media de 778 unidades de frío (Chilling Hours) y Vigo un menor requerimiento con solamente $458 \mathrm{CH}$. En el caso del acúmulo de calor, Ourense presenta el menor requerimiento con una media de 352 GDD (Growth Degree Days) y Vigo alrededor de 450 GDD.
\end{abstract}

Palabras clave. Calor, frío, Ourense, polinización, Quercus, Vigo.

ABSTRACT. Temperature influence on the flowering of Quercus in the South of Galicia (Ourense and Vigo, 1994-98). Plant requirements in order to survive periods of adverse climatic conditions are satisfied by means of physiological adaptions. Thus, woody plants in temperate regions, in order to prevent the water in their cells from freezing, slow their growth down almost to a standstill, entering into a physiological state called "dormancy". Many authors indicate that the end of the dormancy phase require a period of low temperatures followed by another of relatively warm temperatures. When the cold and the heat accumulations are fullfilled, pollination occurs. The objective of this study is to ascertain the influence that a climatic parameter such as temperature exerts on Quercus in the south of Galicia, in order to identify the start of the dormancy period, its duration and the consequents cold and heat requirements that triggers flowering. In this regard, we chose two areas, since they have different temperature patterns, ranging from mild in the case of Vigo to cold in that of Ourense. Monitoring in the two stations was carried out by means of a 7-day LANZONI VPPS 2000 pollen trap, based on the Hirst pollen trap. Quercus began its pollination period in the month of March. In Vigo flowering took place during the first fifteen days and a few days later in Ourense. During the four years under study, in order to overcome the dormancy period, Ourense needed an average of 778 chilling hours $(\mathrm{CH})$ and Vigo had a minor chilling accumulation with only $458 \mathrm{CH}$. In the case of heat accumulation, we found that Ourense had the smallest requirement and Vigo the largest, thus Ourense needed an average of 352 GDD (Growth Degree Days) and Vigo around 450 GDD.

Key words. Chilling, growth degree days, Ourense, polinization, Quercus, Vigo. 


\section{INTRODUCCIÓN}

Las plantas leñosas de floración invernal, después de la fase de crecimiento vegetativo que se produce en la primavera y el verano, presentan durante los meses otoñales una fase en la que ralentizan su crecimiento hasta casi paralizarlo para prevenir el congelamiento del agua intracelular. Los requerimientos necesarios para sobrevivir a condiciones climáticas adversas, son así satisfechos mediante el uso de adaptaciones fisiológicas, entrando en un estado fisiológico denominado "dormancia". Muchos autores definen la dormancia como una suspensión temporal del crecimiento que permite a la planta adaptar su ciclo de crecimiento a la alternancia de las estaciones (Wareing \& Phillips 1978, Kramer \& Kozlowski 1979, Felker y col. 1983, Faust 1989).

Generalmente, el inicio de este período coincide con la disminución del fotoperíodo y del descenso de las temperaturas. En nuestra latitud, estas condiciones tienen lugar durante los primeros momentos del otoño, en los cuales la planta comienza a perder progresivamente las hojas.

Para superar esta fase se necesita un período con bajas temperaturas seguido de otro de temperaturas más elevadas, y solamente después de haber acumulado un cierto número de horas de frío y calor, se desencadena un mecanismo o memoria genética, por el cual la planta se prepara a proseguir su crecimiento en el momento en el que las condiciones ambientales se vuelven favorables (Frenguelli \& Brichi 1998, Felker y col. 1983, Faust 1989).

Es muy difícil identificar el momento en el cuál la planta ha completado sus necesidades y comienza el acumulo de calor que permita reanudar el crecimiento. Un cambio morfológico que tiene lugar durante el período de dormancia es la variación del tamaño del grano de polen, el cual puede ser utilizado como un marcador que nos defina esta fase vegetativa (Felker y col. 1983, Ferranti y col.
1996, Frenguelli y col. 1997, Frenguelli \& Brichi 1998). En las plantas leñosas, la reducción del diámetro de la microspora comienza dos semanas antes del inicio de la dormancia (Frenguelli 1997). El período de plena dormancia es reconocido cuando el volumen de la microspora decrece sobre un $12 \%$ como consecuencia de la deshidratación. Este es un mecanismo de defensa contra las bajas temperaturas, las cuales producen congelamiento dentro de las estructuras reproductoras. Mediante rehidratación, el volumen del grano de polen se incrementa una vez más hasta alcanzar la madurez y en ese momento tiene lugar la antesis. Estas variaciones podrían ser muy útiles para conocer cuando la planta entra y sale de la dormancia.

Es muy importante poder predecir la antesis, sobre todo en el caso de plantas cuyos granos de polen producen polinosis. El objetivo del presente estudio es conocer la influencia que un parámetro climático como la temperatura, ejerce sobre Quercus en el sur de Galicia para llegar a identificar el comienzo del período de dormancia, su duración, y los consecuentes requerimientos de frío y calor para que la floración tenga lugar. En este sentido hemos elegido dos zonas con unos parámetros de temperatura diferentes: una zona templada, Vigo y una fría, Ourense.

\section{MATERIAL Y MÉTODOS}

El inicio del período de polinación de Quercus ha sido estudiado mediante el monitorizaje de las concentraciones atmosféricas de polen en dos zonas del sur de Galicia (Ourense y Vigo), desde el año 1995 al 1998, ambos incluidos (fig. 1).

En el noroeste de España, Ourense se encuentra situada a $130 \mathrm{~m}$ sobre el nivel del mar en el valle de la confluencia de los ríos Miño, Loña y Barbaña, a $90 \mathrm{Km}$ del Océano Atlántico, dentro de la región mediterránea 
subhúmeda de tendencia atlántica. La temperatura media anual es de $14^{\circ} \mathrm{C}$, con una precipitación total anual de $772 \mathrm{~mm}$. Por su parte, la ciudad de Vigo se encuentra emplazada al nivel del mar en la margen derecha de la ría de Vigo. El valle que forma la ciudad, muestra un pequeño sistema de drenaje, principalmente en las direcciones N-S y SW-NE. Presenta un clima marítimo templado, pero en este caso la temperatura media anual es de $14.9^{\circ} \mathrm{C}$, la media de las temperaturas máximas $18.8^{\circ} \mathrm{C}$ y la media de la mínimas de $11^{\circ} \mathrm{C}$. La precipitación total anual es mas elevada, con $1.412 \mathrm{~mm}$.

El monitorizaje ha sido llevado a cabo mediante el uso de un captador volumétrico LANZONI VPPS 2000, desarrollado a partir del modelo de Hirst (Hirst 1952) situado en Ourense en el tejado de la Residencia provincial Sta. María Nai y en Vigo en la terraza del Ayuntamiento.

Los datos meteorológicos empleados para el estudio, fueron registrados por el Instituto Nacional de Meteorología.

El género Quercus está representado por diversas especies en la zona de estudio, destacando cuantitativamente Quercus robur, Quercus pyrenaica, Quercus suber y Quercus rubra (este último utilizado como ornamental). La floración de estas especies se produce de manera secuencial iniciándose con la de Quercus robur, para alrededor de 20 días más tarde producirse la de Quercus pyrenaica y posteriormente Quercus suber. La contribución más importante a la concentración de polen de Quercus a la atmósfera es llevada a cabo por la especie Quercus robur, la cual a su vez es la más abundante en la zona de estudio.

Para obtener el período de polinización se han tenido en cuenta aquellos que incluyen el $98 \%$ y el $95 \%$ del total anual de polen, eliminando el período inicial hasta que la media móvil acumulada de 5 días excede el 1\% (Galán et al., 1995) y el $2.5 \%$ (Andersen 1991).

Con el objeto de comprobar si existe una relación entre las temperaturas previas al inicio
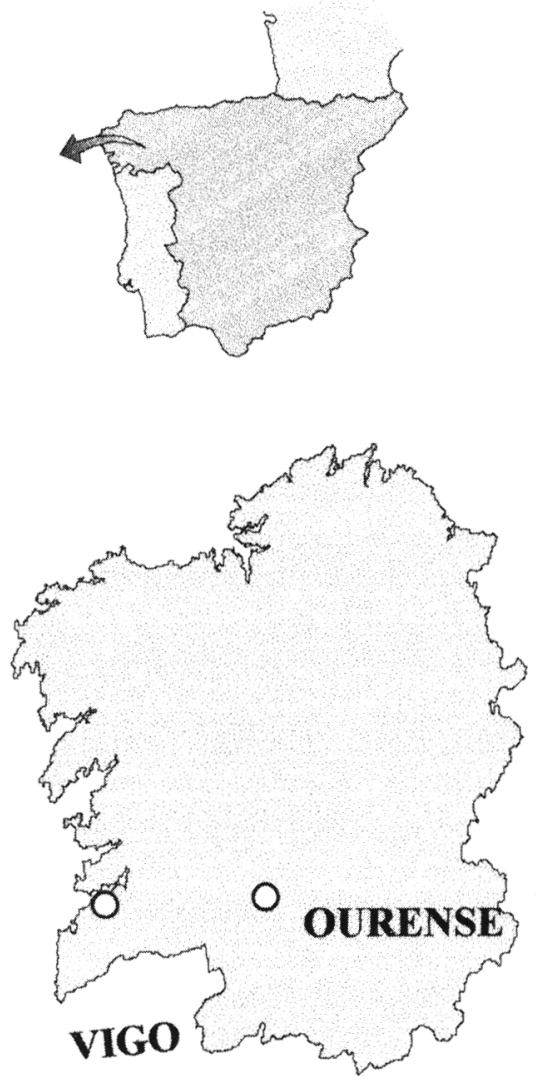

Figura 1. Localización de la zona de estudio. Location of the zone of study.

de la floración y el comienzo de la misma, se ha realizado el test de correlación de Spearman entre el día del comienzo del período de polinización principal y la suma acumulada de las temperaturas medias registradas durante $\operatorname{los} 20,30$ y 40 días anteriores a este.

Los modelos para la realización de la valoración del número de horas de frío para que la planta pueda superar el período de dormancia y de calor hasta producirse la polinización son numerosos (Aron 1983, Linvill 1990, Richardson et al. 1974, Faust 1989, Pires et al. 1997). En el cálculo del requerimiento de frío (Chilling Hours, $\mathrm{CH}$ ) se ha utilizado el modelo propuesto por Aron en 1983, el cual se 
basa en el acúmulo de horas de frío entre 0 y $7.2^{\circ} \mathrm{C}$. Para el presente trabajo se ha comenzado a calcular el acúmulo de horas de frío a partir de las últimas semanas del mes de Octubre, ya que es el momento del año en el cual la temperatura media comienza a ser menor de $12^{\circ} \mathrm{C}$, temperatura que se considera efectiva para que las plantas leñosas de floración invernal comienzan a satisfacer sus requerimientos de frío. Por su parte el acúmulo de calor para inducir la polinización está expresado como GDD (Growth Degree Days en ${ }^{\circ} \mathrm{C}$ ), obtenidos mediante la suma de las temperaturas medias diarias desde la fecha del fin del período frío hasta la fecha en la que se inicia la polinación. A las mismas se les han descontado diferentes temperaturas base de 4 , $4.5,5,5.5$ y $6^{\circ} \mathrm{C}$, las cuales representan $\operatorname{los}$ umbrales sobre los que la temperatura pueda comenzar a ser efectiva para el crecimiento (Faust 1989).

Teniendo en cuenta que la especie Quercus robur es la primera en florecer en la zona, ya que la siguiente inicia su polinación 20 días más tarde, el requerimiento de temperatura para determinar el inicio de la estación polínica de Quercus se refiere exclusivamente a esta, ya que el acúmulo de calor necesario en el caso de las otras sería un poco más elevado.

\section{RESULTADOS}

Para la obtención del período de polinización principal, se ha elegido el descrito por Andersen (1991), el cual incluye el 95\% del polen anual registrado, eliminando el período inicial hasta que la media movil acumulada de 5 días alcanza el $2.5 \%$ del total de polen de Quercus. Al considerar las dos ciudades en conjunto, este método presenta un menor coeficiente de desviación standard en el cálculo de las fechas de inicio de la floración que el descrito por Galán et al. (1995) (tab. 1).

Quercus generalmente inicia su período de polinización en Vigo durante la primera quincena del mes de marzo, mientras que en Ourense esta se produce en la segunda quincena del mes de marzo (figs 2 y 3 ), siguiéndose este patrón de forma casi constante a lo largo de los años de estudio. En Vigo la floración tiene lugar con unos días de adelanto, llegando a ser este de hasta 15 días en el año 1996 (tab. 1). En la misma tabla se puede apreciar que la cantidad

\begin{tabular}{lcccccccc}
\hline \multicolumn{3}{c}{ OURENSE } & \multicolumn{5}{c}{ VIGO } \\
\hline & $\mathbf{1 \%}$ & $\mathbf{2 , 5 0 \%}$ & $\mathbf{F E C H A}$ & POLEN & $\mathbf{1 \%}$ & $\mathbf{2 , 5 0 \%}$ & FECHA & POLEN \\
\hline $\mathbf{1 9 9 5}$ & 82 & 84 & $25-\mathrm{mar}$ & 3333 & 78 & 81 & $21-\mathrm{mar}$ & 3884 \\
$\mathbf{1 9 9 6}$ & 85 & 89 & $31-\mathrm{mar}$ & 2384 & 69 & 75 & $14-\mathrm{mar}$ & 794 \\
$\mathbf{1 9 9 7}$ & 68 & 70 & $11-\mathrm{mar}$ & 2941 & 63 & 66 & $6-\mathrm{mar}$ & 4530 \\
$\mathbf{1 9 9 8}$ & 69 & 73 & $14-\mathrm{mar}$ & 1344 & 51 & 60 & $28-\mathrm{feb}$ & 715 \\
Media & 76 & 79 & & & 65 & 71 & & \\
Minimo & 68 & 70 & & & 51 & 60 & & \\
Maximo & 85 & 89 & & & 78 & 81 & & \\
S.D. & 8,76 & 8,98 & & & 11,32 & 9,33 & & \\
Coef. v.s. & 11,52 & 11,37 & & & 17,36 & 13,23 & & \\
\hline
\end{tabular}

Tabla 1. Día del inicio de la estación polínica a partir del 1 de enero según los métodos del $1 \%$ de Galán et al., 1995 y el $2.5 \%$ de Andersen, 1991 (fecha y número de granos para este último). Starting day of the main pollen season from the first of January, by the methods of the $1 \%$ of Galán et al. 1995 and $2.5 \%$ of Andersen 1991 (date and number of grains for the last). 


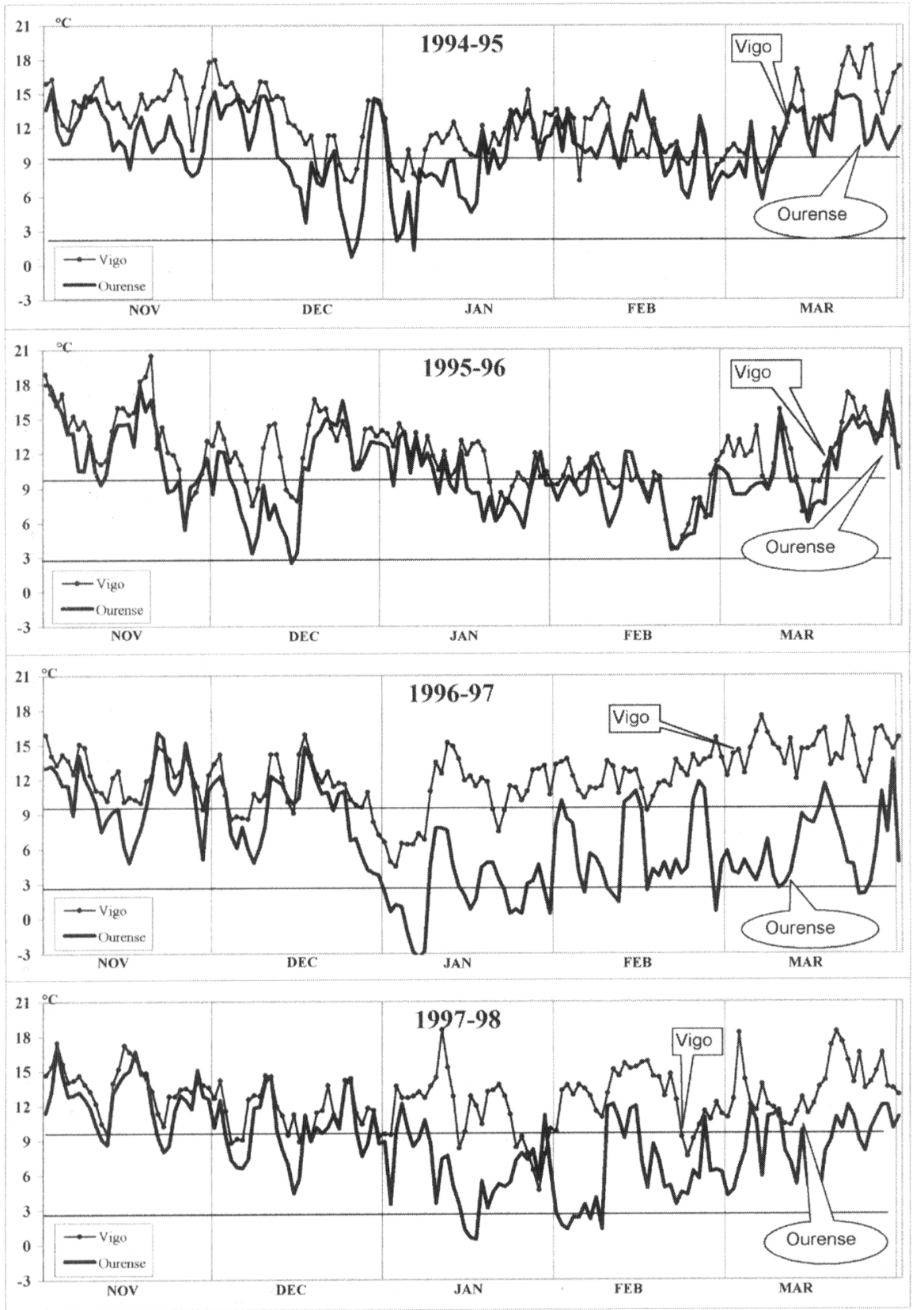

Figura 2. Temperatura media desde Noviembre a Marzo en Vigo y Ourense. Mean temperature from November to March in Vigo and Ourense. 

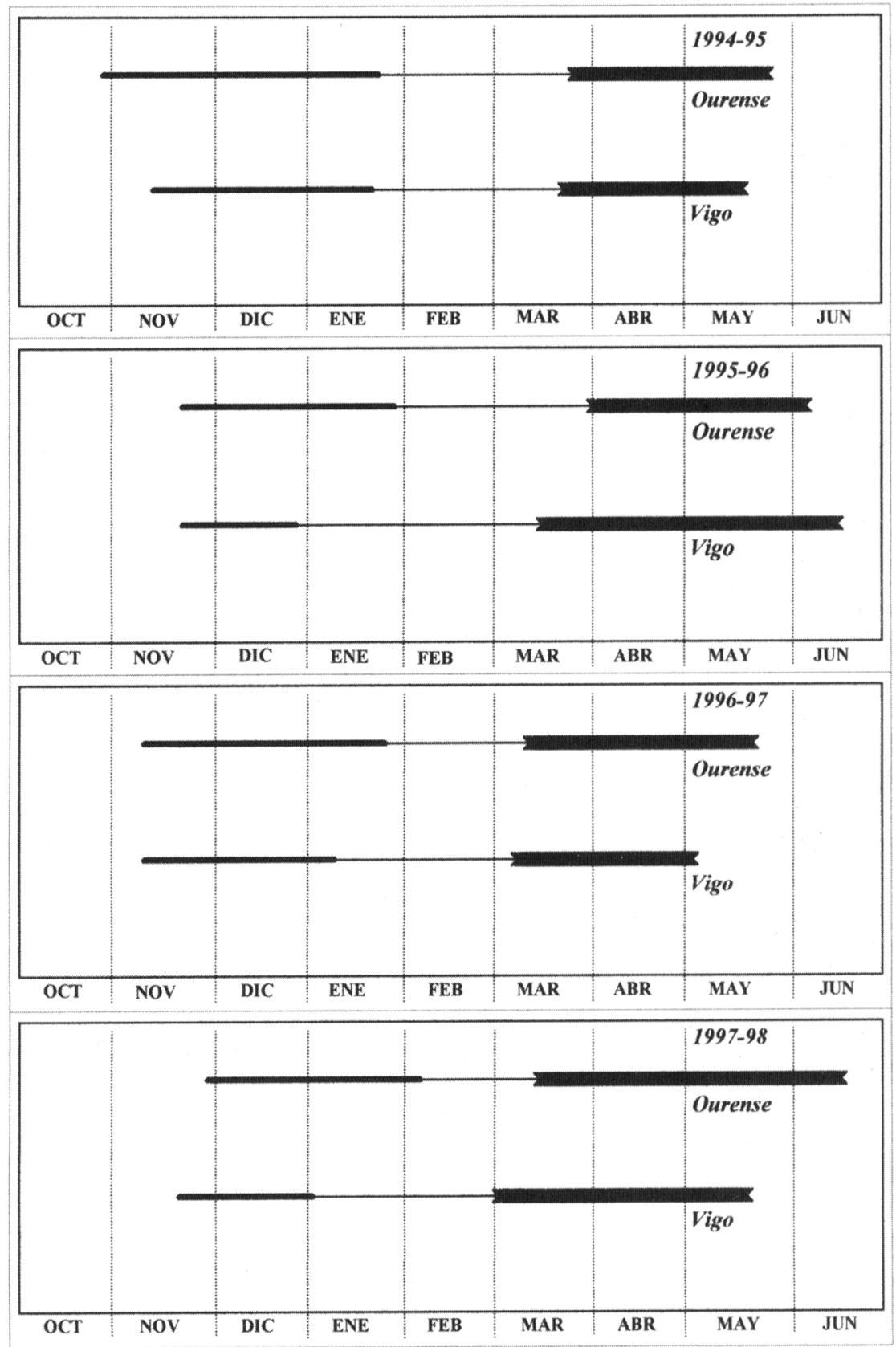

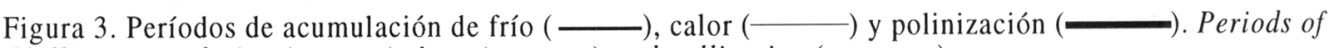
Chilling acumulation (- $\longrightarrow$, heat ( $\longrightarrow$ ) and pollination (-) 
C.U.

G.D.D.

\begin{tabular}{ccccccccccc}
\hline OURENSE & VIGO & & \multicolumn{4}{c}{ OURENSE } & \multicolumn{6}{c}{ VIGO } \\
\hline & & & $\mathbf{4 , 5}$ & $\mathbf{5}$ & $\mathbf{5 , 5}$ & $\mathbf{6}$ & $\mathbf{4 , 5}$ & $\mathbf{5}$ & $\mathbf{5 , 5}$ & $\mathbf{6}$ \\
\hline 770 & 470 & $\mathbf{1 9 9 4 - 9 5}$ & 391 & 360 & 329 & 298 & 440 & 410 & 380 & 350 \\
771 & 433 & $\mathbf{1 9 9 5 - 9 6}$ & 331 & 299 & 267 & 235 & 462 & 425 & 388 & 351 \\
777 & 489 & $\mathbf{1 9 9 6 - 9 7}$ & 341 & 318 & 295 & 272 & 450 & 422 & 394 & 366 \\
792 & 441 & $\mathbf{1 9 9 7 - 9 8}$ & 344 & 324 & 304 & 284 & 447 & 419 & 390 & 362 \\
778 & 458 & Media & 352 & 325 & 299 & 272 & 450 & 419 & 388 & 357 \\
770 & 433 & Mínimo & 331 & 299 & 267 & 235 & 440 & 410 & 380 & 350 \\
792 & 489 & Máximo & 391 & 360 & 329 & 298 & 462 & 425 & 394 & 366 \\
10,15 & 25,94 & S.D. & 26,75 & 25,50 & 25,59 & 27,01 & 9,18 & 6,48 & 5,89 & 7,97 \\
1,31 & 5,66 & Coef. v.s. \% & 7,60 & 7,84 & 8,57 & 9,92 & 2,04 & 1,55 & 1,52 & 2,23 \\
\hline
\end{tabular}

Tabla 2. CH y GDD durante el período de estudio usando diferentes temperaturas base. $C H$ and GDD during period of study by using different base temperatures.

de granos de polen registrados presenta variaciones en las dos ciudades a lo largo de los años de estudio, donde se suceden de forma alternativa años de altas y bajas concentraciones, siendo esto más patente en el caso de la ciudad de Vigo. Este comportamiento bianual es señalado para diversas especies arbóreas y se explicaría por la alternancia en la movilización de las reservas de nutrientes hacia el crecimiento vegetativo o hacia las estructuras reproductoras (Spieksma et al. 1995).

En la tabla 2 se representa la relación entre las temperaturas registradas y la temperatura efectiva para el desarrollo del amento expresada como horas de frío (Chilling Hours). Para ello se ha tenido en cuenta el momento en el cual la temperatura media desciende por debajo de $12.5^{\circ} \mathrm{C}$, temperatura considerada adecuada por Richardson et al. (1974), para iniciar el acúmulo de las horas de frío. Los valores obtenidos fueron diferentes en las dos ciudades, probablemente debido a que Vigo presenta unas temperaturas más cálidas (fig. 2). Durante los cuatro años de estudio, Quercus en Vigo tiene menor requerimiento para superar el período de dormancia (tab. 2), necesitando una media de 458 Chilling Hours. Ourense $788 \mathrm{CH}$, encontrándose en este último caso el coeficiente de desviación standard más bajo con solamente un $1,31 \%$.

La acumulación de calor, expresada como Growth Degree Days $\left(\mathrm{GDD}^{\circ} \mathrm{C}\right)$ que se muestra en la tabla 2, ha sido calculada siguiendo el método de Faust (1989), pero con diferentes temperaturas base de $4.5,5,5.5$ y $6^{\circ} \mathrm{C}$. Los valores obtenidos para ambas ciudades no varían considerablemente y el menor coeficiente de desviación standard se ha obtenido para el caso de la temperatura base de $5,5^{\circ} \mathrm{C}$ para Vigo y $4,5^{\circ} \mathrm{C}$ en Ourense, siendo más constantes el número de $\mathrm{GDD}^{\circ} \mathrm{C}$ en la primera ciudad a lo largo de los años de estudio. Ourense presenta el menor requerimiento de calor con una media de $344 \mathrm{GDD}^{\circ} \mathrm{C}$ y Vigo para que se inicie la floración necesita una media de $390 \mathrm{GDD}^{\circ} \mathrm{C}$.

Finalmente, en la figura 4 , se puede observar que existe una correlación entre la acumulación de horas de frío durante la dormancia y los requerimientos de calor necesarios para que se produzca la polinización, con un valor $\mathrm{r}$ de 0,879 y un valor $\mathrm{p}<0.1$. Cuando se calcula la cantidad de frío necesario, en función de la acumulación de Chilling Hours durante el otoño, se puede apreciar que las 
plantas de la estación más fría acumulan una mayor cantidad de $\mathrm{CH}$, y necesitan una menor cantidad de $\mathrm{GDD}^{\circ} \mathrm{C}$ para el inicio de la floración

Por su parte no se ha encontrado significación al realizar las correlaciones entre las fechas de comienzo del período de polinización con el número de Chilling Hours y con el número de Growth Degree Days, a lo largo de los años de estudio.

\section{DISCUSIÓN}

Muchos autores (Frenguelli et al. 1991, Richardson et al. 1974, Spieksma et al. 1989) describen que la estacionalidad de la polinización es debida a importantes y sucesivas necesidades de temperatura. En las ciudades objeto de este estudio se ha encontrado una correlación estadísticamente significativa entre el día del comienzo del período de polinización principal y la suma acumulada de las temperaturas medias registradas durante $\operatorname{los} 20,30$ y 40 días anteriores a la floración con un valor $\mathrm{p}<0.01$ en la mayoría de los casos (tab. 3). Estos datos muestran una significación lo suficientemente fuerte como para llegar a la conclusión de que la temperatura ejerce una gran influencia sobre el inicio de la floración en el sur de Galicia. En este sentido y para obtener una mayor información hemos estudiado los requerimientos de frío y de calor que Quercus necesita para romper el período de dormancia que sufre en los meses previos al inicio de la floración.

El inicio de la floración de Quercus en el sur de Galicia se produce con un ligero adelanto frente a otras localidades del noroeste peninsular (Aira et al. 1998). Las diferencias climáticas de las zonas de estudio, el que los requerimientos de frío y de calor puedan estar determinados genéticamente y que en cada zona

\begin{tabular}{|c|c|c|c|c|}
\hline TEMPERATURAS & Fechas de inicio del P.P.P. & $R$ & $R^{2}$ & $P$ \\
\hline Media $T^{a}$ MEDIA & 2-decadas precedentes & 0.496 & 0.246 & 0.101 \\
\hline \multirow[t]{2}{*}{ VIGO } & 3-decadas precedentes & 0.510 & 0.260 & 0.091 \\
\hline & 4-decadas precedentes & 0.642 & 0.412 & 0.024 \\
\hline Media Tª MAXIMA & 2-decadas precedentes & 0.691 & 0.477 & 0.022 \\
\hline \multirow[t]{2}{*}{ VIGO } & 3-decadas precedentes & 0.429 & 0.184 & 0.164 \\
\hline & 4-decadas precedentes & 0.211 & 0.045 & 0.510 \\
\hline Media Ta MINIMA & 2-decadas precedentes & 0.367 & 0.134 & 0.241 \\
\hline \multirow[t]{2}{*}{ VIGO } & 3-decadas precedentes & 0.433 & 0.188 & 0.160 \\
\hline & 4-decadas precedentes & 0.522 & 0.273 & 0.081 \\
\hline Media T ${ }^{a}$ MEDIA & 2-decadas precedentes & 0.949 & 0.902 & 0.050 \\
\hline \multirow[t]{2}{*}{ OURENSE } & 3-decadas precedentes & 0.896 & 0.802 & 0.104 \\
\hline & 4-decadas precedentes & 0.914 & 0.834 & 0.086 \\
\hline Media Ta MAXIMA & 2-decadas precedentes & 0.912 & 0.833 & 0.087 \\
\hline \multirow[t]{2}{*}{ OURENSE } & 3 -decadas precedentes & 0.908 & 0.824 & 0.092 \\
\hline & 4-decadas precedentes & 0.975 & 0.951 & 0.025 \\
\hline Media T ${ }^{a}$ MINIMA & 2-decadas precedentes & 0.264 & 0.069 & 0.736 \\
\hline \multirow[t]{2}{*}{ OURENSE } & 3-decadas precedentes & 0.729 & 0.531 & 0.271 \\
\hline & 4-decadas precedentes & 0.809 & 0.656 & 0.190 \\
\hline
\end{tabular}

Tabla 3. Correlaciones entre la media de las temperaturas de los 20, 30 y 40 días anteriores al inicio del período de polinización y el día de inicio del período de polinización principal. Correlations between the average of the temperatures of the 20,30 and 40 days before the start of the pollen season and the starting day of the main pollen season. 
los árboles pertenezcan a poblaciones genéticas diferentes, son las causas que pueden explicar los retrasos en la fecha de inicio del período de polinización. En este sentido es una constante a lo largo de los años que la floración se produzca antes en la ciudad de Vigo, localidad que presenta las temperaturas más cálidas.

Si estudiamos tanto el número de días en los que se producen los acúmulos de frío y calor como la cantidad de CH y GDD necesarias para alcanzarlos, se observa que los lugares con temperaturas más frías presentan un mayor requerimiento de frío durante un mayor número de días y un menoy acúmulo de calor durante un menor número de días, ya que en estas zonas el árbol necesita protegerse durante más tiempo.

Este patrón de comportamiento se cumple en nuestro caso si observamos una localidad más fría como es Ourense frente a otra más cálida como Vigo. En las figuras 2 y 3 se observa como Ourense acumula una mayor cantidad de frío durante un mayor número de días y posee asimismo un menor requerimiento de calor, tanto en lo que concierne al menor número de días necesarios para alcanzar los requerimientos de calor, como a que estos son cuantitativamente menos importantes (tab. 2).

A nivel particular, y dentro de cada zona, las diferencias en el inicio de la floración a lo largo de los años de estudio se pueden explicar tanto por una alta temperatura durante el período de dormancia como por una baja temperatura durante el tiempo de acúmulo de calor.

El caso de Vigo (fig. 2), debido a las bajas temperaturas registradas en los momentos del acúmulo de calor durante 1995 y 1996, se necesitaron un número de días superior al de otros años para satisfacer las necesidades, 10 que provocó un ligero retraso en las fechas del
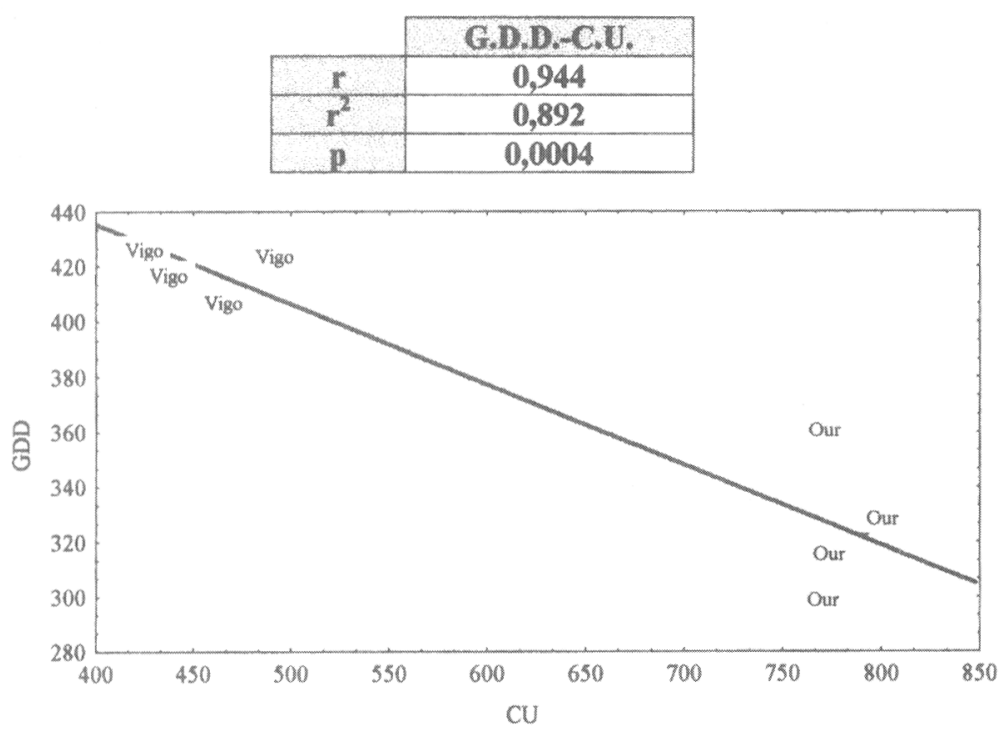

REGRESIÓN LINEAR G.D.D.-C.U.

$y=552.327-0.292^{*} x+$ eps

Figura 4. Correlación y regresión entre el acúmulo de horas de frío y calor durante el período de dormancia de Quercus. Correlation and regression between chilling hours and growth degree days during the period of dormancy of Quercus. 
inicio de la floración (en especial en 1996 donde se necesitaron además un mayor número de GDD). En 1997 se adelantó el inicio del período de polinización, debido a que fue el año con las temperaturas más elevadas en los momentos previos a la floración, concretamente durante el mes de febrero.

En el invierno del año 1994 el número de días en los que se ha acumulado frío es más elevado debido a las altas temperaturas registradas durante la primera quincena del mes de Diciembre. En los inviernos del resto de los años se ha producido el proceso contrario.

Finalmente, los resultados obtenidos al realizar la recta de regresión entre la acumulación de $\mathrm{CH}$ frente a los GDD y representados en la figura 4 refuerzan la afirmación anterior de que los lugares con temperaturas más frías presentan un mayor requerimiento de frío y un menor acúmulo de calor. En un extremo de la recta de regresión se encuentran los puntos que corresponden a los valores alcanzados durante los años de estudio en Vigo y en el extremo opuesto los de Ourense.

Se ha intentado encontrar la posible correlación entre las fechas de comienzo del período de polinización con el número de Chilling Hours y con el número de Growth Degree Days, para así poder conocer cual de estos dos parámetros posee una mayor influencia frente a la fecha del inicio de la floración. No se ha encontrado significación lo cual parece indicar que además de la temperatura existen otros parámetros como puede ser el fotoperíodo, horas de sol, humedad, etc que influyen en el proceso.

\section{CONCLUSIONES}

En las ciudades del sur de Galicia estudiadas, Quercus inicia su período de polinización principal durante el mes de marzo, generalmente en Vigo durante la primera quincena y en Ourense durante la segunda.
A lo largo de los cuatro años de estudio para finalizar el período de dormancia en Ourense se necesita una media de 778 Chilling Hours, presentando Vigo la menor necesidad de frío con solamente $458 \mathrm{CH}$ En el caso de la acumulación de calor, Ourense necesita un menor requerimiento y Vigo mayor, con una media de 344 y 390 GDD respectivamente.

Nuestros datos muestran que las diferencias que se presentan con respecto al inicio de las estaciones polínicas de Quercus en la zona templada y en diferentes regiones climáticas, pueden ser explicadas mediante las temperaturas del aire registradas durante los períodos que preceden el inicio de la estación polínica. Así, las localidades con temperaturas más frías presentan un mayor requerimiento de frío durante un mayor número de días y un menor acúmulo de calor en menos días, ya que en estas zonas el árbol necesita protegerse durante más tiempo.

\section{BIBLIOGRAFÍA}

AIRA, M.J., A. DOPAZO M.V. JATO y F.J. RODRÍGUEZ - 1998- Aerobiología de Galicia: Estación de Santiago de Compostela (1997). Rea 4: 107-110.

ANDERSEN, T.B. -1991- A model to predict the beginning of the pollen season. Grana 30: 269275.

ARON, R. -1983- Availability of chilling temperatures in California. Agric. Meteor. 28: 351-363.

FAUST, M. -1989- Physiology of temperate zone fruit trees. In JhonWiley \& Sons, New York, pp. 188-189.

FELKER, F.C., H. A. ROBITAILLE \& F. D. HESS -1983- Morphological and ultrastructural development and starch accumulation during chilling of sour cherry flower buds. Amererican Journal of Botany 70(3): 376-386.

FERRANTI, F., R. ANDREUTTI, E. TEDESCHINI \& G. FRENGUELLI - 1996- Microsporogenesis and dormancy period in some arboreal species. Giorn. Bot. Ital. 130: 319 
FRENGUELLI, G., F. TH. M. SPIEKSMA, E. BRICCHI, B. ROMANO, G. MINCIGRUCCI, A.H. NIKKELS, W. DANKAART \& F. FERRANTI -1991- The influence of air temperature on the starting dates of the pollen season of Alnus and Populus. Grana 30: 196200.

FRENGUELLI, G. -1997- Pollen volume change as a marker of the dormancy period in some arboreal plants. Allionia 35: 181-187

FRENGUELLI, G., F. FERRANTI, E. TEDESCHINI \& R. ANDREUTTI -1997-Volume changes in pollen grain of Corylus avellana L. (Corylaceae) during development. Grana 36: 289-292.

FRENGUELLI, G. \& E. BRICCHI -1998- The use of the pheno-climatic model for forecasting the pollination of some arboreal taxa. Aerobiologia 14: 39-44.

GALÁN, C., J. EMBERLIN, E. DOMÍNGUEZ, R. BRYANT \& F. VILLAMANDOS - 1995- A COMPARATIVE ANALISIS OF DAILY VARIATIONS IN THE GRAMINAE POLLEN COUNTS AT CORDOBA, SPAIN AND LONDON, UK. GRANA 34: 189-198.

HIRST, J. -1952- An automatic volumetric sporetrap. Ann. Appl. Biol. 36: 257-265.

KRAMER, P.J. \& T.T. KOZLOWSKI -1979Phisiology of woody plants. Academic Press New York, 546-627.

LINVILL, D. - 1990- Calculating chilling hours and chilling units from daily maximum and minimum temperature observations. Hortscience 25 (1): 14-16.

PIRES, J., N.A., VILLA \& H. SILVEIRA - 1997- A model including photoperiod in degree days for stimating Hevea bud growth. Int. J. Biometeorol 41:1-4.

RICHARDSON, E.A., D.S. SCHUYLER \& D.W WALKER -1974- A model to for estimating the completion of rest of "Redhaven" and "Elberta" peach trees. Hortoscience 9(4): 331-332.

SPIEKSMA, F. TH. M., G. FRENGUELLI, A.H. NIKKELS, G. MINCIGRUCCI, L.O.M.J. SMITHUIS, E., BRICCHI, W. DANKAART \& B. ROMANO -1989- Comparative study of airborne pollen concentrations in central Italy and The Netherlands (1982-1985). Grana 28: 25-36.

SPIEKSMA, F. TH. M., J. EMBERLIN, M. HJELMROOS, S. JÄGER \& R.M. LEUSCHNER
-1995- Atmospheric birch (Betula) pollen in Europe: trends and fluctuations in annual quantities and the starting dates of the seasons. Grana 34 (1): 51-57.

WAREING, P.F. \& I.D.J. PHILLIPS -1978- The control of growth and differentiation in plants. Pergamon press, Oxford, 253-280.

Aceptado para su publicación en julio de 2000

Dirección de los autores. Dept. de Biología Vegetal y Ciencias del Suelo. Facultad de Ciencias de Ourense. Campus As Lagoas. E-32004 Ourense (España). 